\title{
BARRIERS TO THE ADOPTION OF THE ART APPROACH AS PERCEIVED BY DENTAL PRACTITIONERS IN GOVERNMENTAL DENTAL CLINICS, IN TANZANIA
}

\author{
Emil N. KIKWILU ${ }^{1}$, Jo E. FRENCKEN ${ }^{2}$, Jan MULDER ${ }^{3}$
}

\author{
1- DDS, Mdent, Department of Preventive and Community Dentistry, School of Dentistry, Muhimbili University of Health and Allied Sciences, Dar es \\ Salaam, Tanzania. \\ 2- DDS, MSc, PhD, Department of Global Oral Health, Radboud University Nijmegen Medical Centre, College of Dental Sciences, Nijmegen, the \\ Netherlands. \\ 3- MSc, Department of Preventive and Restorative Dentistry, Radboud University Nijmegen Medical Centre, College of Dental Sciences, Nijmegen,
} the Netherlands.

Corresponding address: J.E. Frencken - Department of Global Oral Health, Radboud University Nijmegen Medical Centre, College of Dental Sciences - P.O Box 9101 - 6500 - HB Nijmegen, the Netherlands - e-mail: j.frencken@dent.umcn.nl

Received: October 8, 2008 - Accepted: January 10, 2009

\begin{abstract}
bjectives: This study aimed to determine the magnitude of the barriers to the practice of Atraumatic Restorative Treatment (ART) as perceived by dental practitioners working in pilot dental clinics, and determine the influence of these barriers on the practice of ART. Material and Methods: A validated and tested questionnaire on barriers that may hinder the practice of ART was administered to 20 practitioners working in 13 pilot clinics. Factor analysis was performed to generate barrier factors. These were patient load, management support, cost sharing, ART skills and operator opinion. The pilot clinics kept records of teeth extracted; teeth restored by conventional approach and teeth restored by ART approach. These treatment records were used to compute the percentage of ART restorations to total teeth treated, percentage of ART restorations to total teeth restored and percentage of total restorations to total teeth treated. The mean barrier scores were generated and compared to independent variables, using the t-test. The influence of barriers to ART-related dependent variables was determined using Pearson correlation coefficients. Results: Mean barrier values were low, indicating low influence on ART practice. Female practitioners had higher scores on patient load than male practitioners $(p=0.003)$. Assistant Dental Officers had higher scores on cost sharing than Dental Therapists $(p=0.024)$. Practitioners working in urban clinics had higher mean scores on patient load than those who worked in rural clinics $(p=0.0008)$. All barrier factors were negatively correlated with ART practice indices but all had insignificant association with ART practice indices. Conclusion: The barriers studied were of low magnitude, with no significant impact on practice of ART in dental clinics in the pilot area.
\end{abstract}

Key words: Atraumatic Restorative Treatment. Adoption. Barriers. Government dental clinics. Tanzania.

\section{INTRODUCTION}

In 2000, the WHO Regional Office for Africa issued a policy document recommending that all African countries should train district oral health workers in Atraumatic Restorative Treatment (ART) for managing dental caries ${ }^{20}$. In response to this, an ART demonstration project was started, training 30 dental practitioners who were working in 16 governmental dental clinics. Starting with a demonstration project was in line with the proposals of van Palenstein Helderman, et al. ${ }^{19}$ (2003), who suggested that a small-scale demonstration project was needed to assess the effectiveness, efficacy and sustainability of the Basic Package of Oral Care (BPOC) or its components before a large-scale or countrywide introduction was initiated. Since
ART is both a preventive and a restorative technique, the demonstration project was started after baseline studies were conducted. These investigated barriers to restorative care as perceived by dental patients ${ }^{5}$, and dental practitioners ${ }^{6}$. Dental practitioners' attitudes, subjective norms and intentions to practice ART were also assessed ${ }^{7}$. These studies included "diagnostic analysis" to identify factors likely to influence the introduction of ART, as suggested in the Effective Health Care Bulletin ${ }^{18}$, and by Oxman, et al. ${ }^{13}$ (1995).

The findings of the baseline studies were used for choosing appropriate interventions from a range of interventions that had been shown to be effective ${ }^{1,16}$. These included holding a 7-day ART training course for the 30 dental practitioners and supplying each with a starter pack 
of glass-ionomer cement and ART hand instruments. Other interventions were: routinely informing patients about the availability of ART as a new restorative approach, twiceyearly supervisory visits to all dental practitioners in their dental clinics, and one follow-up meeting at the end of each year of the follow-up period. The order for additional and routine supplies of glass-ionomer cement and ART hand instruments was also initiated at the Medical Stores Department of the Ministry of Health and Social Welfare, through the office of the Chief Dental Officer. The supervisory visits and the follow-up meetings were both used to identify and solve the barriers that were negatively affecting the smooth practice of ART.

Barriers that were identified and addressed during the follow-up period included undue delays in the ordering of glass-ionomer and ART hand instruments by the Medical Store Department of the Ministry of Health and Social Welfare. These delays caused some clinics to run out of glass-ionomer supplies, thereby frustrating the ART introduction process. Other barriers identified during the supervisory visits were related to patient management. Most practitioners were focusing on the management of patients' presenting symptoms and not at all on the teeth and consequently, practitioners were leaving cavities suitable for ART untreated. As Philips, et al. ${ }^{14}$ (2001) pointed out; this problem is inherent in traditional medical/dental education and practice, which focus on relieving symptoms. Also, in most clinics, practitioners treated to patients needing extraction first, and those requiring restorative and preventive care were left to wait until all extractions had been handled. This disadvantaged those patients who would have liked to have their teeth restored, so many opted for extraction. Another identified barrier was cost-sharing, because in some clinics the fee for a restoration was higher than the cost of an extraction. Efforts to address this issue were initiated. During all supervisory visits clinical ART skills were emphasised, to improve practitioners' selfconfidence and reduce any professional uncertainty that could lead to disempowerment ${ }^{4}$.

Although obstacles were identified and addressed through the built-in checks, using group discussions and supervisory visits, it was not clear whether all the obstacles had been adequately identified and addressed because of the weaknesses inherent in group discussions ${ }^{8}$. As Craig, et $\mathrm{al}^{3}{ }^{3}$ (2002) pointed out; using focus group discussions has disadvantages, in the sense that discussants may not raise all issues on their minds. Therefore a false scenario of barrier identification was a possibility. An additional possibility was the promotion of a uniformity of views through group dynamics ${ }^{18}$. To complement the methods used in identifying obstacles through group discussion and visits, a self administered questionnaire was constructed, to facilitate identification of barriers that might have not been adequately addressed during the follow-up period.

The questionnaire took into the account the 6 constraints that may hinder the smooth implementation of an innovation requiring a change in clinical dental practice, as summarized by McGlone, et al. ${ }^{11}$. These include patient influence and opinion, attitude and knowledge of practitioners, dentists' feelings regarding self esteem and conscience, the organisational and social environment of the practice setting, funding arrangements and undergraduate and postgraduate education. A questionnaire taking these 6 constraints into account had been used in South Africa, with high reliability coefficients $^{12}$.

The aims of this study were: a) to determine the magnitude of the barriers, as perceived by dental practitioners working in pilot dental clinics and b) to establish the influence of these barriers on the practice of ART in pilot dental clinics.

\section{MATERIAL AND METHODS}

\section{Questionnaire}

A questionnaire previously used to assess the factors that inhibited the implementation of the ART in South Africa ${ }^{12}$ was used in the present study, with minor modifications to suit the Tanzanian situation (Figure 1). The South African version assessed 7 aspects of care. These included patient load, availability of supplies, competence of chair-side assistant, oral health management, operator opinion, patients' opinions, and skills for practicing ART. A question regarding supplies was removed from the questionnaire used in Tanzania because this barrier item had already been identified and addressed adequately during the follow-up meetings and supervisory visits. It was replaced by a question related to cost-sharing, identified during the supervisory visits as a potential barrier to the practice of ART.

Six positive and negative statements were constructed for each aspect of care. This was done to improve the internal consistency of the questionnaire. Only the question about the chair-side assistant covered 3 items. Therefore, a total of 39 item statements were included in the final questionnaire. These were phrased in such a way as to allow a respondent to rank his/her degree of agreement to each according to a 5-point Lickert scale ranging from $1=$ strongly disagree to $5=$ strongly agree. In addition to the 39 items, 3 demographic characteristics of practitioners were recorded. These were clinic location, gender, and qualification.

\section{Clinic Treatment Records}

Written approval for this study was obtained from the Ethical Committee of the Muhimbili University College of Health Sciences, by a letter referenced MU/RP/AEC/VOL. II/130. Treatment records for extracted teeth, teeth restored by conventional methods, and teeth restored by the ART approach were collected from 13 of the 16 clinics, which had complete data covering the entire 31-month follow-up period.

\section{Subjects}

At the commencement of the ART pilot project 30 dental practitioners were working in these dental clinics. After 31 months of follow-up, 9 practitioners were no longer practising in these clinics, and one practitioner could not 
Personal characteristics:

1. Clinic ..........; 2. Age [...]; 3. Year of graduation [..]; 4. Sex (1. Male; 2. Female);

5. Qualification: (1. DDS, 2. ADO, 3. DT)

In the following questions, insert the number corresponding to the degree to which you agree to each statement provided. The options are: 1. Strongly disagree; 2. Disagree; 3. Undecided; 4. Agree; 5. Strongly agree, 0. Not applicable

\section{Patient load}
a. I have to treat many patients needing tooth extractions each working day [ ]
b. I make ART restorations to all carious lesions suitable for ART I see in my clinic [ ]
c. Due to many patients needing tooth extraction, I can not make as many ART restorations as I wish [ ]
d. Tooth extraction takes all of my working time in my clinic [ ]
e. I have time to make at least two ART restorations in my clinic [ ]
f. Overall, the patient load for urgent oral care in my clinic is high [ ]

\section{Operator opinion}

a. I feel better when I do not have to give a local anaesthetic [ ]

b. I feel a sense of accomplishment when I am able to restore a tooth [ ]

c. I would like to spend more time rendering ART in my clinic [ ]

d. Having experience with drilling as well as ART, it is generally better to restore teeth using drill than ART [ ]

e. I still have some doubts on the effectiveness of ART restorations [ ]

f. Overall, I am proud of the ART skills I have acquired [ ]

\section{Patient opinion}

a. In my clinic, patients prefer tooth extraction to restorations [ ]

b. My patients are very grateful and satisfied, if I restore their teeth using the ART technique [ ]

c. My patients are very grateful and satisfied, when I don't have to inject them [ ]

d. My patients are very grateful and satisfied, when I don't have to drill their teeth [ ]

e. My patients doubt the effectiveness of ART restorations [ ]

f. Overall, my patients like ART restorations [ ]

\section{Oral health service management}

a. I have experienced some negative responses regarding ART from my superiors[ ]

b. Hospital management suspect that ART will tarnish the reputation of the hospital [ ]

c. Hospital management feel that glass ionomer is too expensive for the hospital to afford [ ]

d. The hospital management is pleased that I make ART restorations [ ]

e. Members of the hospital management are willing to be treated by ART [ ]

f. Overall, my hospital administration fully support ART [ ]

10. Clinical ART skills

g. I have difficulties in deciding as to whether a tooth is for ART restoration or extraction [ ]

h. I feel comfortable when excavating carious lesion for ART restoration [ ]

i. I feel comfortable mixing glass ionomer cement [ ]

j. I feel comfortable applying the glass ionomer cement mix into the cleaned cavity [ ]

k. I feel comfortable when removing excess cement to adjust for occlusion [ ]

I. Overall, I have adequate skills to make ART restorations [ ]

\section{Chair side assistance}

m. I have trained my assistant to assist me when making ART restorations [ ]

n. My assistant is skilled to assist me in rendering ART [ ]

o. Having to make ART restorations without assistant affects my efficiency [ ]

\section{Cost sharing}

a. I treat few cases with ART because many of my patients cannot afford to pay for ART restorations [ ]

b. Most patients I see in my clinic accept to pay for ART restorations [ ]

c. I have in many occasions failed to treat patients using ART because of patients lack money to pay for a restoration [ ]

d. If I want to make an ART restoration and a tooth extraction on same sitting, many patients cannot afford to pay for both treatments [ ]

e. If the fee for ART was reduced, I would treat more patients with ART restorations[ ]

f. Overall, the fee for ART restorations is prohibitive for majority of patients to access ART restorations [ ]

FIGURE 1- Questionnaire used in the present study 
fill in the form, owing to administrative duties. The remaining 20 dental practitioners completed the questionnaire, which was administered within the 31-month follow up mark.

\section{Construction of variables}

Factor analysis was performed for each question to obtain the communalities of the items studied. Cronbach's alpha of 0.5 was set as the cut-off point for a factor construction. In this analysis, the questions regarding patient opinion and chair-side assistant were removed from subsequent analysis because they had a Cronbach's alpha below 0.5. The remaining 5 questions had Cronbach's alphas ranging from 0.68 to 0.86 . These formed the 5 factor barriers: patient load, management support, cost sharing, clinical ART skills and operator opinion and were used as dependent variables in subsequent analyses.

The primary and permanent dentition treatments rendered in each clinic were recorded. A dependent variable, total treatment, was constructed by summing up the extracted teeth + conventionally restored teeth + ART-restored teeth for both dentitions. Other dependent variables were: pctART-all as a percentage ratio of ART restorations to total treatment rendered; ART-fraction as a percentage ratio of ART restorations to total tooth restorations (ART restorations + conventional restorations); and pct-totalrest as a percentage ratio of total restorations to total treatments rendered.

Independent variables considered in this study were: gender, qualification \{Dental Officer (DO), Assistant Dental Officer (ADO), Dental Therapists (DT) $\}$ and clinic location (urban, rural). Qualification DO had only one record. Since the total dental training programme for DO and ADO is the same (5 years) and their skills were considered comparable, it was agreed to pool the data for $\mathrm{DO}$ and $\mathrm{ADO}$ in $\mathrm{ADO}$ in the subsequent analyses.

\section{Statistical Analysis}

The data were then transferred to SAS program (SAS Institute Inc., Cary, NC, USA) for analysis by a statistician. The mean for pct-ART-all; ART-fraction; pct-totalrest and for the five factor barriers was calculated. Pearson correlation coefficients between factor barriers and pct-ARTall; ART-fraction; pct-totalrest were generated to identify possible relationships. The t-test was performed to identify differences in perception of barrier factors between gender, qualification and clinic location. The effect of the barrier factors on the practice of ART were tested using Pearson correlation coefficients between barrier factors and 3 ARTrelated dependent variables (pct-ART-all; ART-fraction; pcttotalrest). The level of statistical significance was set at 5\%.

\section{RESULTS}

In total one Dental Officer, 13 Assistant Dental Officers and 6 Dental Therapists filled in the questionnaire. Table 1 shows the mean value and standard error of the barrier factors by gender, qualification and clinic location. The mean values for all barrier factors were low; below score 3. Female practitioners rated patient load barrier factor higher than male practitioners did $(\mathrm{p}=0.003)$. Assistant Dental Officers (ADO) rated the cost sharing barrier factor higher than Dental Therapists did (DT) $(\mathrm{p}=0.024)$. Practitioners in urban clinics rated the patient load barrier factor higher than practitioners in rural clinics rated it $(\mathrm{p}<0.001)$.

Table 2 summarizes the correlation coefficients between barrier factors and 3 measures of the contribution of the ART restorations to treatment rendered. With the exception of operator opinion, all barrier factors had a negative correlation with the percentage ART restorations to total treatment and with percentage ART restorations to total restorations. Nevertheless, none of the studied barrier factors had a significant association with the ART-related dependent variables.

TABLE 1- Mean and standard error (se) values of barrier factors by gender, qualification and clinic location

\begin{tabular}{|c|c|c|c|c|c|c|c|c|c|c|}
\hline \multirow[b]{2}{*}{ Barrier } & \multirow{2}{*}{$\begin{array}{l}\text { Total } \\
\text { Mean } \\
\text { (se) }\end{array}$} & \multicolumn{2}{|c|}{ Gender } & \multirow[b]{2}{*}{ p-value } & \multicolumn{2}{|c|}{ Qualification } & \multicolumn{4}{|c|}{ Clinic location } \\
\hline & & $\begin{array}{l}\text { Male } \\
\text { Mean } \\
(\mathrm{se})\end{array}$ & $\begin{array}{l}\text { Female } \\
\text { Mean } \\
(\mathrm{se})\end{array}$ & & $\begin{array}{l}\text { ADO } \\
\text { Mean } \\
(\mathrm{se})\end{array}$ & $\begin{array}{l}\text { DT } \\
\text { Mean } \\
\text { (se) }\end{array}$ & p-value & $\begin{array}{c}\text { Rural } \\
\text { Mean } \\
\text { (se) }\end{array}$ & $\begin{array}{l}\text { Urban } \\
\text { Mean } \\
\text { (se) }\end{array}$ & p-value \\
\hline Patient load & $2.6(0.2)$ & $2.4(0.2)$ & $3.3(0.2)$ & 0.003 & $2.6(0.2)$ & $2.9(0.4)$ & 0.531 & $2.2(0.1)$ & $3.0(0.1)$ & 0.001 \\
\hline Management & & & & & & & & & & \\
\hline support & $2.2(0.1)$ & $2.2(0.1)$ & $2.2(0.1)$ & 0.711 & $2.2(0.1)$ & $2.2(0.1)$ & 0.764 & $2.2(0.1)$ & $2.2(0.1)$ & 0.777 \\
\hline Cost sharing & $2.1(0.2)$ & $2.1(0.2)$ & $2.3(0.3)$ & 0.697 & $2.4(0.2)$ & $1.7(0.2)$ & 0.024 & $2.0(0.3)$ & $2.2(0.3)$ & 0.640 \\
\hline ART skills & $1.7(0.1)$ & $1.6(0.1)$ & $1.9(0.1)$ & 0.053 & $1.7(0.1)$ & $1.7(0.1)$ & 0.908 & $1.6(0.1)$ & $1.7(0.1)$ & 0.673 \\
\hline Operator opinion & $1.4(0.1)$ & $1.3(0.1)$ & $1.4(0.2)$ & 0.718 & $1.5(0.1)$ & $1.1(0.1)$ & 0.059 & $1.4(0.4)$ & $1.4(0.1)$ & 0.872 \\
\hline
\end{tabular}

Scale for barriers: $1=$ strongly disagree, $2=$ disagree, $3=$ undecided, $4=$ agree, $5=$ strongly agree

ADO: Assistant Dental Officer, DT: Dental Therapist. 
TABLE 2- Mean barrier values, standard error (se), Cronbach's $\alpha$ reliability coefficients for barrier factors and Pearson correlation coefficients and their associated p-values between barrier score and ART contribution

\begin{tabular}{lccccc} 
Barrier & Mean & Cronbach's $\boldsymbol{\alpha}$ & $\begin{array}{l}\text { ART contribution } \\
\text { pct-ART-all }\end{array}$ & pct-totalrest & ART-fraction \\
& $(\mathbf{s e})$ & & & & \\
\hline Patient load & $2.6(0.2)$ & 0.68 & $r=-0.21(p=0.43)$ & $r=-0.17(p=0.52)$ & $r=-0.04(p=0.88)$ \\
Management support & $2.2(0.1)$ & 0.76 & $r=-0.40(p=0.11)$ & $r=0.31(p=0.23)$ & $r=-0.45(p=0.07)$ \\
Cost sharing & $2.1(0.2)$ & 0.79 & $r=-0.48(p=0.05)$ & $r=-0.04(p=0.88)$ & $r=-0.38(p=0.13)$ \\
ART skills & $1.7(0.1)$ & 0.86 & $r=-0.27(p=0.30)$ & $r=-0.03(p=0.92)$ & $r=-0.39(p=0.12)$ \\
Operator opinion & $1.4(0.1)$ & 0.72 & $r=0.32(p=0.23)$ & $r=-0.17(p=0.51)$ & $r=0.05(p=0.85)$ \\
\hline
\end{tabular}

\section{DISCUSSION}

Interpretation of the findings of the present study should be made with the following limitations in mind. Firstly, the questionnaire did not deal with issues related to a broad range of incentives. Those covered in the questionnaire were limited to satisfaction which the practitioner may derive from making a painless restoration and/or having the ability to restore teeth without even a drilling machine. Secondly, the study was undertaken in a clinic situation where restorative care was negligible, so few people demanded restorations. This may have resulted in a false impression of the barriers, which could become important if more people demand such a service. Therefore, more barriers might become evident if the ART approach becomes more familiar to more people to the extent of raising the demand for ART restorations. In such a situation, barriers like clinical inertia ${ }^{2,15}$ and remuneration structures ${ }^{9,10,17}$ may arise. These could, in turn, raise the scores for patient load and operator opinion. Thirdly, patient opinion and chair-side assistant were removed from further analysis, because of their low reliability coefficients. Therefore, their effects could not be measured. Nevertheless, the individual item scores ranged between 1 and 2, indicating that they were not important barriers to the practice of ART under the current oral care circumstances in Tanzania.

The findings that the mean values for the barriers investigated in the present study were low indicated that these barriers were perceived by practitioners as having little influence on the use of ART to restore teeth. The mean barrier scores obtained in the present study were similar to those reported in relation to dental practitioners in South Africa ${ }^{12}$. However, whereas barriers in this study had no statistically significant correlation with the percent ART restorations to total treatment rendered, in South Africa all barriers had a significant negative association with percent ART restorations to total treatment rendered ${ }^{12}$.

Although no barrier factor had a significant negative correlation with ART contribution to total treatment, the borderline significance for cost-sharing and management support indicated their great potential for affecting the practice of ART, and that these barrier factors were not adequately addressed during the process evaluation. Cost- sharing was identified as a barrier to the ART practice, as many patients could not afford to pay the fee for a restoration. Discussions between the hospital authorities had been concluded in favour of reviewing the fee for restoration downwards. However, at the time of data collection, the decision to lower the fee for restorative care had not been effected. Because management support was mainly based on the attitudes of the hospital management towards dentistry, its significance as a barrier was difficult to address fully within the short follow-up period. This barrier was also identified in the study on barriers to restorative care as perceived by dental practitioners in Tanzania ${ }^{6}$. There is still a need to identify means of improving the image of dentistry among some hospital managers in Tanzania.

The negative correlations in the present study, between the barriers investigated and the contribution of ART restorations to total treatment rendered, indicated that these were actual barriers and exerted a negative influence on ART practice. Operator opinion, on the other hand, had positive correlation with the contribution of ART restorations to total treatment rendered. This indicated that the opinion of dental practitioners had no negative influence on the practice of ART. Operator opinion, the attitude of the operator towards ART practice, had been shown to have no influence on the intention to practice ART in an earlier study in Tanzania ${ }^{7}$.

\section{CONCLUSIONS}

Barriers studied were of low magnitude, with no significant impact on the practice of ART in dental clinics in the pilot area.

\section{ACKNOWLEDGEMENTS}

The authors wish to thank the dental practitioners for their time they spent to respond to the questionnaire. 


\section{REFERENCES}

1- Bero LA, Grilli R, Grimshaw LM, Harvey E, Oxman AD, Thomson MA. Closing the gap between research and practice: an overview of systematic reviews of interventions to promote the implementation of research findings. The Cochrane Effective Practice and Organization of Care Review Group. BMJ. 1998;317:465-8.

2- Cabana MD, Rand CS, Powe NR, Wu AW, Wilson MH, Abboud PA, et al. Why don't physicians follow clinical practice guidelines? A framework for improvement. JAMA. 1999;282:1458-65.

3- Craig N, McGregor S, Drummond N, Fischbacher M, Iliffe S. Factors affecting the shift towards a "primary care-led" NHS: qualitative study. Br J Gen Pract. 2002;52:895-900.

4- Kai J, Beavan J, Faull C, Dodson L, Gill P, Beighton A. Professional uncertainty and disempowerment responding to ethnic diversity in health care: a qualitative study. PLoS Med. 2007;4(11):e323.

5- Kikwilu EN, Frencken JE, Mulder J, Masalu JR. Barriers to restorative care as perceived by dental patients attending government hospitals in Tanzania. Community Dent Oral Epidemiol. 2009;37:35-44.

6- Kikwilu EN, Frencken JE, Masalu JR, Mulder J. Barriers to restorative care as perceived by dental practitioners in Tanzania. Community Dent Health. In press 2009.

7- Kikwilu EN, Frencken JE, Mulder J, Masalu JR. Dental practitioners' attitudes, subjective norms and intentions to practice Atraumatic Restorative Treatment (ART) in Tanzania. J Appl Oral Sci. 2009;17(2):97102 .

8- Kitzinger J. Qualitative research: Introducing focus groups. Br Med J. 1995;311:299-302.

9- Kristiansen IS, Mooney G. The general practitioner's use of time: is it influenced by the remuneration system? Soc Sci Med. 1993;37:393-9.

10- Kristiansen IS, Mooney G. Remuneration of GP services: time for more explicit objectives? A review of the systems in five industrialized countries. Health Policy. 1993;24:203-12.

11- McGlone P, Watt R, Sheiham A. Evidence-based dentistry: an overview of the challenges in changing professional practice. Br Dent $\mathrm{J}$. 2001;190:636-9.

12- Mickenautsch S, Frencken JE, van't Hof MA. Factors inhibiting the implementation of the Atraumatic Restorative Treatment approach in public oral health services in Gauteng province, South Africa. J Appl Oral Sci. 2007;15:1-8.

13- Oxman AD, Thomson MA, Davis DA, Haynes RB. No magic bullets a systematic review of 102 trials of interventions to improve professional practice. Can Med Assoc J. 1995;153:1423-31

14- Philips LS, Branch WT, Cook CB, Doyle JP, El-Kebbi IM, Gallina DL, et al. Clinical inertia. Ann Intern Med. 2001;135:825-34

15- Rindal DB, Rush WA, Boyle RG. Clinical inertia in dentistry: a review of the phenomenon. J Contemp Dent Pract. 2008;9:113-21.

16- Siddiqi K, Newell J, Robinson M. Getting into practice: what works in developing countries? Int J Qual Health Care. 2005;17:447-54.

17- Sturmberg JP. Implementing best practice guidelines: the influence of personal characteristics. J Eval Clin Pract. 1999;5:223-6.

18- The University of York. Effective health care: getting evidence into practice. Bulletin of the effectiveness of health service interventions for decision makers. 1999;5(1).
19- van Palenstein Helderman W, Lo E, Holmgren C. Guidance for the planning, implementation and evaluation of oral health care demonstration projects for under-served populations. Int Dent J. 2003;53:19-25.

20- WHO-AFRO - World Health Organization. Regional Office for Africa. Oral health in the African Region: a regional strategy 1999-2008. Brazzaville: WHO/AFRO; 2000 